ARTICLE

DOI: $10.1038 / \mathrm{s} 41467-018-05391-2$

\title{
Pairwise library screen systematically interrogates Staphylococcus aureus Cas9 specificity in human cells
}

Josh Tycko (1,5, Luis A. Barrera1,6, Nicholas C. Huston ${ }^{1,7}$, Ari E. Friedland', Xuebing Wu², Jonathan S. Gootenberg $\mathbb{D}^{3}$, Omar O. Abudayyeh ${ }^{4}$, Vic E. Myer ${ }^{1}$, Christopher J. Wilson (D) ${ }^{1}$ \& Patrick D. Hsu ${ }^{1,8}$

Therapeutic genome editing with Staphylococcus aureus Cas9 (SaCas9) requires a rigorous understanding of its potential off-target activity in the human genome. Here we report a highthroughput screening approach to measure SaCas9 genome editing variation in human cells across a large repertoire of 88,692 single guide RNAs (sgRNAs) paired with matched or mismatched target sites in a synthetic cassette. We incorporate randomized barcodes that enable whitelisting of correctly synthesized molecules for further downstream analysis, in order to circumvent the limitation of oligonucleotide synthesis errors. We find SaCas9 sgRNAs with 21-mer or 22-mer spacer sequences are generally more active, although high efficiency 20-mer spacers are markedly less tolerant of mismatches. Using this dataset, we developed an SaCas9 specificity model that performs robustly in ranking off-target sites. The barcoded pairwise library screen enabled high-fidelity recovery of guide-target relationships, providing a scalable framework for the investigation of CRISPR enzyme properties and general nucleic acid interactions.

\footnotetext{
${ }^{1}$ Editas Medicine, 11 Hurley St., Cambridge, MA 02141, USA. ${ }^{2}$ Whitehead Institute for Biomedical Research, Cambridge, MA 02142, USA. ${ }^{3}$ Department of Systems Biology, Harvard, Cambridge, MA 02138, USA. ${ }^{4}$ Department of Health Sciences and Technology, Massachusetts Institute of Technology, Cambridge, MA 02139, USA. ${ }^{5}$ Present address: Department of Genetics, Stanford University School of Medicine, Stanford, CA 94305, USA. ${ }^{6}$ Present address: Arrakis Therapeutics, 35 Gatehouse Dr., Waltham, MA 02451, USA. ${ }^{7}$ Present address: Department of Molecular Biophysics and Biochemistry, Yale University, New Haven, CT 06511, USA. ${ }^{8}$ Present address: Laboratory of Molecular and Cell Biology, Salk Institute for Biological Studies, La Jolla, CA 92037, USA. Correspondence and requests for materials should be addressed to C.J.W. (email: christopher.wilson@editasmed.com)

or to P.D.H. (email: patrick@salk.edu)
} 
T he compact SaCas9 enables in vivo delivery of Cas9 and multiple single guide RNAs (sgRNA) packaged within a single adeno-associated virus (AAV) vector ${ }^{1,2}$, serving as a promising platform for gene editing therapies. AAV-SaCas9 is capable of achieving therapeutic levels of genome editing in preclinical animal models of Duchenne muscular dystrophy ${ }^{3,4}$, ornithine transcarbamylase deficiency ${ }^{5}$, and of HIV infection ${ }^{6}$. Translating these promising initial results into medicines requires a rigorous understanding of intended and unintended genome editing. SaCas9-mediated off-target effects have been detected with genome-wide methods, including GUIDE-seq ${ }^{7}$ and BLESS $^{1,8}$, and direct visualization of dSaCas9-EGFP binding in cells ${ }^{9}$. However, the sequence determinants of SaCas9 cleavage specificity have not been profiled. Furthermore, SaCas9 is known to efficiently cleave genomic DNA with spacer lengths from 20 to $24 \mathrm{nt}^{1,2}$, but the effect of spacer length on specificity is not known.

To systematically interrogate SaCas9 specificity in human cells, we developed a method to test a library of sgRNAs against a library of genome-integrated synthetic target sequences. Lentiviral delivery of the pairwise library cassette results in integration of a sgRNA and paired synthetic target site in the genome. We designed a library of 88,692 guide-target pairs, distributed among 73 sgRNA groups. Within a group, the sgRNA had shared sequence in positions 1-18 and ranged in length from 19 to $24 \mathrm{nt}$ spacers. All sgRNAs were paired with target sites bearing all possible single mismatches and subsets of sgRNAs were paired with all possible double mismatches or all possible double transversions. Five groups of sgRNA were paired with target sites bearing all possible single insertions and deletions, to study the effect of DNA and RNA bulges. The protospacer adjacent motif (PAM) was held constant at $5^{\prime}$-CAGGGT- $3^{\prime}$ to match the consensus sequence of $5^{\prime}$-NNGRRT- $3^{\prime}{ }^{1}$. This pairwise library design enables high-throughput characterization of SaCas9 in cells, while controlling for the effects of delivery and chromatin context, and allows us to determine the optimal spacer lengths for specific genome editing.

\section{Results}

Double barcode design improves pairwise screen measurements. Lentiviral delivery of the pairwise library cassette results in integration of a sgRNA and paired synthetic target site in the genome (Fig. 1a). We designed a library of 88,692 guide-target pairs, distributed among 73 sgRNA groups (Supplementary Table 1). We measured the genome editing activity of these guide-target pairs in human cells (Fig. 1b). The library cassette lentivirus was transduced in HEK 293FT cells at low multiplicity-of-infection (MOI) to enrich for single-copy integration events, ensuring independent editing reactions per cell. Genomic DNA was extracted 0, 3, and 14 days after SaCas9 transduction and the library cassette was PCR-amplified prior to Illumina sequencing.

After editing has occurred, insertions or deletions (indels) in the target site can obscure the original guide-target pair relationship. Accordingly, each library member (i.e., a unique guide-target pair) was linked with a unique, error-correcting Hamming barcode ${ }^{10} 16$ basepairs downstream of the cut site (Supplementary Fig. 1A). Post-editing, this Hamming barcode identifies the original guide-target sequence in a sequence read. The indel frequencies associated with each Hamming barcode determine editing efficiency for each guide-target pair. Across sequencing runs, we found a perfect match to a Hamming code in $83 \%$ of reads, on average. After using the error-correcting algorithm to retrieve the missing Hamming codes, we were able to match $23 \%$ of the remaining reads to a Hamming code, resulting in $\sim 87 \%$ Hamming codes being recovered in total.
Reasoning that a subset of molecules representing a particular library member would be subject to synthesis errors that generate an inappropriate mismatch or indel, we appended an additional randomized barcode $(\mathrm{rBC})$ downstream of the sgRNA. These $\mathrm{rBC}$ effectively barcode unique lentiviral integrations. Sequencing from Day 0 was used to whitelist the guide-target cassettes that were error-free and sufficiently represented prior to Cas9 delivery. This whitelist minimizes false positive indels that arise from synthesis errors or other causes, and improves the reproducibility of pairwise library screens by filtering out library members with insufficient cellular representation (i.e., library members with a low number of rBCs).

Indel rates and the corresponding off:on-target ratios at Day 3 and 14 were then computed using only whitelisted rBCs (i.e., cassettes that were error-free at Day 0). Indel levels were observed to be increasingly reproducible as the minimum number of unique whitelist $\mathrm{rBCs}$ per library member was also increased (Fig. 1c). After filtering for library members with at least 20 independent integrations (i.e., $\geq 20 \mathrm{rBCs}$ ), $83 \%$ of the library remained and the biological replicates were correlated $\left(R^{2}=0.76\right)$ (Fig. 1d).

21 and 22 nt spacers are most efficient. We first analyzed the ontarget activity of SaCas9. Based on our observation of saturation in indel levels by Day 6 in a pilot time-course study (Supplementary Fig. 1), we sequenced the screen samples on Day 3 to determine representative but non-saturated indel efficiency, and again on Day 14.21 and $22 \mathrm{nt}$ spacers edited on-target sites most efficiently at both time points (Fig. 1e and Supplementary Fig. 2A). This result refines smaller-scale studies (of 4 and 21 sgRNAs) that had defined the optimal spacer length as being $21-23 \mathrm{nt}^{1}$ or $20-24 \mathrm{nt}^{2}$.

20 nt spacers are less tolerant of mismatches. Next, we compared the average off-target indel rates for mismatched guidetarget library members and found targets with single mismatches were edited 3.4-fold less than matched targets on average $(p<$ 0.01, Dunn's multiple comparisons test) (Fig. 1f), while the targets with double mismatches or bulges were edited even less frequently.

SaCas9 off:on-target activity can be further considered as a function of both mismatch identity and position. Overall, mismatch tolerance was low in the $\sim 9$ nt PAM-proximal seed region, oscillated between positions 13-19, and was higher at the PAM-distal region (Fig. 2a, b). This tolerance pattern was consistent across spacer lengths, but the $21 \mathrm{nt}$ spacer was the most tolerant of mismatches at any position. The $20 \mathrm{nt}$ spacer was significantly less tolerant of single mismatches than the 21 or 22 nt spacer at both Day 3 and Day $14(p<0.01$, Dunn's multiple comparisons test, Fig. 2a and Supplementary Fig. 2B).

We considered that the apparent differences in specificity across spacer lengths might be due to the differences in guide efficiency. To study specificity while controlling for only highly active guides, we examined the top ten most active guides within each spacer length group. We did not observe a statistically significant difference between the on-target efficiencies of the top ten guides of the 20,21, and $22 \mathrm{nt}$ spacer groups (Dunn's multiple comparisons test, Supplementary Fig. 3A), although the $20 \mathrm{nt}$ spacer efficiency did trend lower than the $21 \mathrm{nt}$ spacer efficiency. In this subset, guides with a $20 \mathrm{nt}$ spacer were again less tolerant of single mismatches than the 21 or $22 \mathrm{nt}$ spacer groups at both Day 3 and Day $14(p<0.001$, Dunn's multiple comparisons test, Supplementary Fig. 3B). Notably, these highly active $20 \mathrm{nt}$ spacers are atypical, but this analysis shows that their lower tolerance of mismatches cannot be attributed to low overall activity. 
a

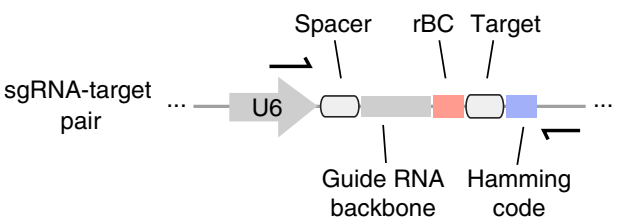

C

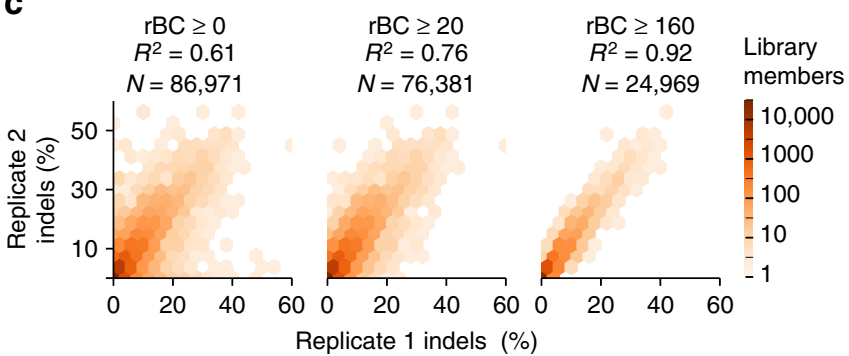

d

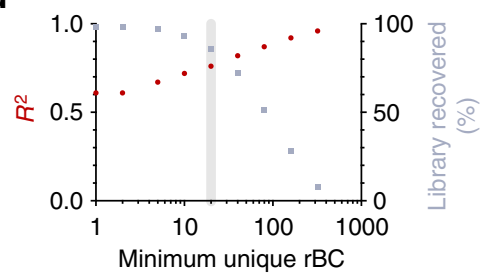

b

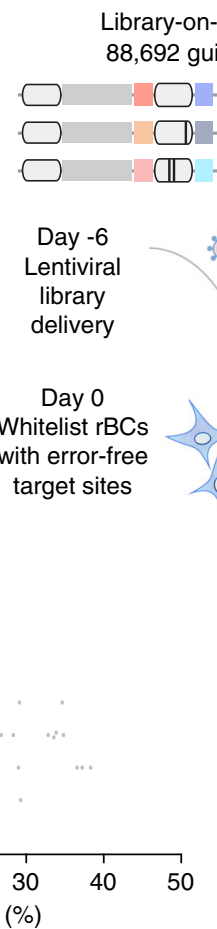

Day 3 and 14

Measure indels on reads

with whitelist rBCs

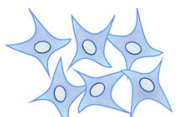

Day 0

Lentiviral

SaCas9

delivery

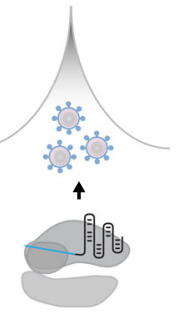

f

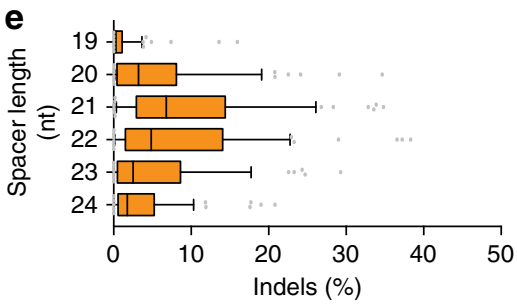

Fig. 1 Pairwise library screen of SaCas9 genome editing specificity. a Schematic of the pairwise library cassette. Individual library members have variable spacer and target sequences, and each member is identifiable by a unique $15 \mathrm{nt}$ error-correcting Hamming barcode. Individual molecules of each library member are tagged with a unique randomized barcode $(r B C)$. b Schematic of the pooled pairwise library screen workflow. Each guide-target pair is associated with many rBCs. The library was initially installed in HEK 293T cells by lentivirus and sequenced to generate a whitelist of rBCs associated with error-free guide-target pairs. SaCas9 was then delivered by lentivirus. After 3 and 14 days, the library was sequenced to measure Cas9-mediated indels. c Reproducibility of a pairwise library screen increases if a greater number of whitelist $\mathrm{rBC}$ is required for each library member. Heat color represents the number of library members in that hexagonal bin, while white area represents 0 library members. $\mathbf{d}$ The fraction of recovered library members decreases as a greater number of $\mathrm{rBC}$ is required. All downstream analyses were performed with a minimum of 20 unique whitelist rBCs for each library member (grey). e On-target indel efficiency on Day 3 for SaCas9 guide-target pairs, binned by spacer length. ( $n=653$ guide-target pairs). $\mathbf{f}$ Comparison of SaCas 9 activity across categories of target sites. The scrambled targets are negative controls ( $n=47,374$ guide-target pairs). $\mathbf{c}$ through $\mathbf{f}$ use data from Day 3 postSaCas9 delivery. Boxes in $\mathbf{e}$ and $\mathbf{f}$ denote median and interquartile range (IQR), and whiskers extend to the 10th and 90th percentile

Across the full set of spacers, the $20 \mathrm{nt}$ spacer was also less tolerant of double mismatches than the longer guides $(p<0.0001$, Dunn's multiple comparisons test, Supplementary Fig. $2 \mathrm{C}$ ), in a position-dependent manner (Fig. 2c, e). 21 nt spacers had a mean $16 \%$ off:on target activity ratio while we observed a mean of only $2 \%$ off:on-target activity across all double-mismatched sites targeted by $20 \mathrm{nt}$ spacers (Fig. 2c, d), at Day 3. We observed an increase in double-mismatch tolerance at Day 14, and the $20 \mathrm{nt}$ spacer remained significantly less tolerant than the longer guides $(p<0.0001$, Dunn's multiple comparisons test, Supplementary Fig. 2C).

Upon inspecting the subset of library members with bulges, (single-nucleotide indels in the target site), we found that both RNA and DNA bulges in the sgRNA:target duplex were minimally tolerated at positions $1-18$, regardless of sgRNA length (Supplementary Fig. 4B, C). This bulge intolerance remained consistent on Day 14. However, PAM-distal bulges were near-completely tolerated. The bulge nucleotide identity had no significant effect on indel measurements (Supplementary Fig. 4B, C).

Validation of spacer length affecting specificity. Given the effect of the spacer length on mismatch tolerance, we conducted Northern blot analysis to confirm that this range of spacer lengths is reliably maintained in human cells. sgRNA from 18 to $24 \mathrm{nt}$ were accurately maintained at full length when expressed from a
U6 promoter, regardless of whether or not the $5^{\prime}$ nucleotide was a guanine (Supplementary Fig. 5). Further, sgRNA were hardly detectable in cells lacking SaCas9 expression.

To validate these findings with an orthogonal method, we designed GFP-targeting sgRNAs with variable length spacers and synthesized sgRNA with single mismatches at every position. In independent wells, we transiently transfected $\mathrm{SaCas} 9$ and each sgRNA into a stable HEK 293T-GFP cell line and measured GFP knockout by flow cytometry. Consistent with the screen, we observed low tolerance of PAM-proximal mismatches and more variable PAM-distal tolerance, in a mismatch identity and nucleotide position-dependent manner. As in the screen, we again found $20 \mathrm{nt}$ spacers to be less tolerant of single mismatches (Supplementary Fig. 6).

SaCas9 specificity score ranks off-target sites. We next formulated a non-linear regression specificity score, trained from our screen dataset, to integrate the relative contribution of mismatch position, identity, number, and density. These scores correlate well with the observed off-target activity in the screen (Supplementary Fig. 7). Our model assumes that mismatches can be modeled independently and performs similarly on off-targets with single or multiple mismatches (Supplementary Fig. 7). This scoring algorithm was capable of predicting SaCas9 specificity in the orthogonal GFP mismatch assay with high performance (Fig. 3a). Furthermore, the score performed well in ranking 
a
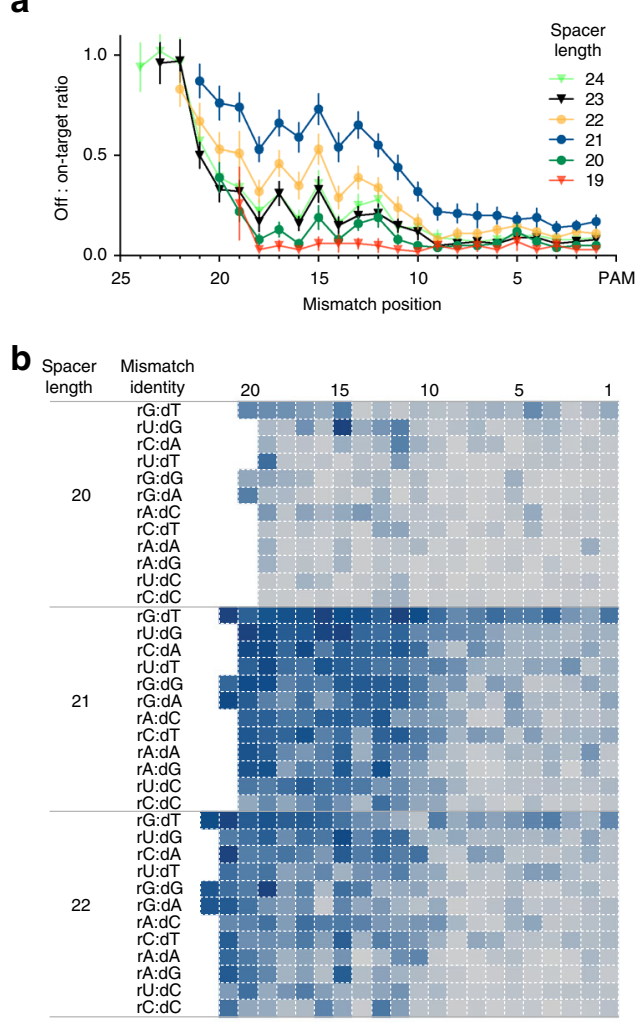

$c$

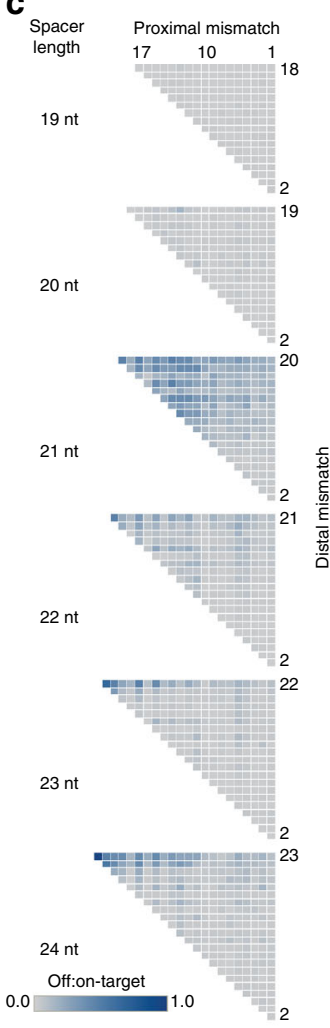

d

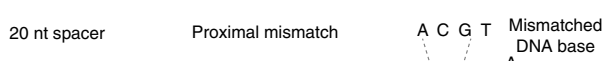
20 nt spacer Proximal mismatch A C G T Mismatched $1817161514131211109876654030210 . A$

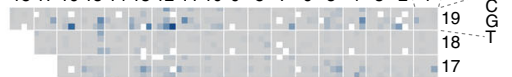

$$
\text { . }
$$

.

Fig. 2 SaCas 9 sgRNA with shorter spacers are less tolerant of mismatches. a Average effect of sgRNA spacer length and mismatch position on SaCas9 single-mismatch tolerance. Mean $\pm 95 \%$ confidence interval is shown $(n=16,742$ guide-target pairs). b Heatmap of relative SaCas 9 cleavage efficiency for each possible RNA:DNA base pair, calculated for all single mismatch library members ( $n=10,344$ guide-target pairs). c Heatmap of relative SaCas 9 double-mismatch tolerance across different spacer lengths and positions. Data is aggregated from $10 \mathrm{sgRNAs}$ for which all possible single and double mismatches were tested ( $n=28,707$ guide-target pairs). d DNA base identity and position effect on double-mismatch tolerance for 20 nt spacer sgRNA ( $n=6017$ guide-target pairs) and e for $21 \mathrm{nt}$ spacer sgRNA ( $n=6729$ guide-target pairs). All panels use data from Day 3 post-SaCas9 delivery
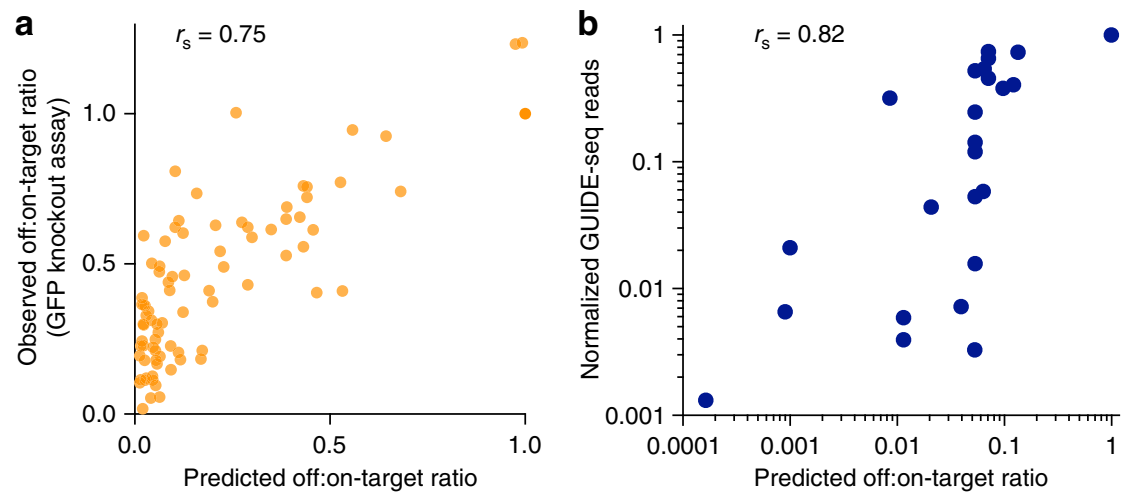

Fig. $3 \mathrm{SaCas} 9$ specificity model ranks synthetic and endogenous off-target sites. a A regression model trained on the pairwise library screen data correlates with an independent validation set ( $n=86$ guide-target pairs). The sgRNAs knock out a stable GFP and include single mismatches in the spacers to mimic off-target effects. b Spearman rank correlation of the SaCas 9 specificity model compared with previously reported SaCas 9 GUIDE-seq data from a different cell type, $\mathrm{U}^{2} \mathrm{OS}^{7}$

endogenous off-target sites in a different cell type (U2OS), as measured by GUIDE-seq ${ }^{7,11}$, with a Spearman correlation of 0.82 (Fig. 3b).

\section{Discussion}

These findings support a simple strategy to mitigate the risk of off-target activity by adjusting the spacer length. $20 \mathrm{nt}$ spacers reduce SaCas9 mismatch tolerance, which parallels the finding that 17-18 nt truncated guide RNA spacers can improve SpCas9 specificity ${ }^{12-15}$. However, these shorter spacers are less efficient on average so more experimental screening may be necessary to find a suitably efficient $20 \mathrm{nt}$ spacer. This strategy can be combined with expanded PAM variants in situations where the number of candidate guides is $\mathrm{low}^{7}$, such as for targeting of pathogenic SNPs. Our study supports known strategies 
such as selecting sgRNAs with maximal sequence dissimilarity from off-target sites and avoiding off-target sites with only PAMdistal mismatches. Importantly, the specificity model trained on SaCas9-specific parameters can be used for in silico selection of guides and to prioritize off-target sites for follow-up.

The challenge of characterizing genome editing and nucleic acid specificity is well-suited to high-throughput approaches because of the large space of possible guide-target pairs. While genome-wide off-target detection methods ${ }^{1,11,13,16-19}$ are important for characterizing individual sgRNA of interest, they are also limited by the availability of endogenous off-target sites, and do not provide general models of specificity ${ }^{20}$. A complementary approach is to generate a synthetic library that more thoroughly covers the space of possible off-target sites. To date, such studies have primarily been performed in vitro ${ }^{21,22}$ and in yeast ${ }^{23}$. While specificity of the Cas9 from $S$. pyogenes has been previously profiled in human cells via cell surface marker knockout and flow cytometry ${ }^{24}$, the pairwise library approach described here provides a more programmable, alternative method. Previous reports suggest that even single-nucleotide changes in a sgRNA can strongly affect activity by changing RNA secondary structure ${ }^{25}$, but our screen avoids this concern and mimics a real-world scenario by placing the mismatches in the target DNA. Further, the edits are directly measured at the DNA sequence. This property enables high-throughput evaluation of nuclease-mediated DNA repair outcomes.

A related approach was recently employed to characterize Cpf1 as a genome editing tool ${ }^{26}$ by screening linked libraries, but was sometimes limited by high error rates at baseline. In contrast, our design includes two barcodes, one for the target-guide pair and another to track each integration event, which allows us to whitelist the targets that were error-free at baseline and recover the majority of the library for analysis. Low MOI delivery of our pairwise library further facilitates the measurement of independent editing reactions for each guide-target pair compartmentalized in individual cells.

Our barcoded pairwise library screening approach provides a general framework for understanding and engineering nucleic acid interactions, and could be exploited for oligonucleotide probe or switch design. We demonstrate its utility via highthroughput characterization of SaCas9 specificity, which could be extended to interrogate other nuclease properties in future studies.

\section{Methods}

Library design and cloning. Custom Python scripts were written for the pairwise library design. Initially, we generated a random library of 19-24 nt spacer sequences. To minimize undesired Cas9 targeting outside the lentivirally-integrated pairwise library cassette, the sgRNA sequences were then computationally optimized to be highly orthogonal to the human reference genome by filtering the list of candidate spacers against the hg19 assembly. A 5' G was held constant for every unique sgRNA spacer for reliable U6-driven expression. The (PAM) was held constant at 5'-CAGGGT-3'. The number of single-mismatch, double-mismatch, DNA bulge, RNA bulge, and control guides that were generated for each unique sgRNA spacer is described in Supplementary Table 1.

Hamming codes were generated using a modified version of a Python script available on Github (https://github.com/mdshw5/hamstring). We modified the code to increase the barcode length to $15-\mathrm{bp}$, expanding the number of available barcodes so as to cover the whole library. These barcodes are composed of ten data bases, four checksum bases, and one parity base. We excluded homopolymers of $>3 \mathrm{nt}$ and filtered for GC content from $30 \%$ to $70 \%$. This resulted in 812,547 barcodes, which were sub-sampled to 93,000 , enabling an increased edit-distance greater than 2 .

The library was synthesized as a pool of single-stranded 135-142 nt oligonucleotides by CustomArray. Variable oligo length is due to the varied spacer lengths. In order to accommodate constraints of synthesis length, oligo synthesis did not include the full length sgRNA tail, but instead included a short BsmBI Type IIS tracrRNA cloning site in between the spacer and target sequences.

First, the library oligo was PCR amplified and cloned by Gibson Assembly (NEB) into the pairwise library lentiviral backbone, which included a U6 promoter for sgRNA expression and a puromycin resistance gene (Supplementary Data 1 and Supplementary Data 2). The plasmid was then electroporated into Endura ElectroCompetent cells (Biorad). To maintain library complexity, the transformed cells were plated on large $245 \times 245 \mathrm{~mm}$ LB plates (Teknova) and colony density was estimated by serially diluting and spreading the transformed cells on LB plates. Colonies were quantified with an online image analysis tool (Benchling).

This pre-tracrRNA pairwise library was sequenced by MiSeq to verify its representation and rate of synthesis errors. The tracrRNA was synthesized as a PAGE-purified ultramer (Integrated DNA Technologies), with an additional $8 \mathrm{nt}$ $5^{\prime}$-NNNNNNNN-3' rBC at the $3^{\prime}$ end to identify independent DNA molecules representing each library member. The tracrRNA-rBC oligo was PCR amplified and ligated into the BsmBI cloning site of the pre-tracrRNA. The resulting plasmid pool was also subjected to deep sequencing by MiSeq to verify library member representation and synthesis quality. The library sequences are available (Supplementary Data 3).

Cell culture. HEK293T (ATCC, CRL-3216) and HEK293FT (Life Technologies, catalog \#R700-07) cells were cultured in Dulbecco's modified eagle medium (DMEM), supplemented with $10 \%$ fetal bovine serum (FBS) and $1 \%$ penicillinstreptomyocin (D10). Cells were maintained in T225 flasks while screening. The antibiotic selections used $10 \mu \mathrm{g} / \mathrm{ml}$ blasticidin or $0.5 \mu \mathrm{g} / \mathrm{ml}$ puromycin. HEK293GFP (GenTarget, catalog \#SC001) cells were maintained in DMEM, supplemented with $10 \%$ FBS, $5 \%$ penicillin-streptomycin, and $2 \mathrm{mM}$ Glutamax. All HEK293 were kept at $37^{\circ} \mathrm{C}$ in a $5 \% \mathrm{CO}_{2}$ incubator. These cell lines were used due to their efficient transfection and lentiviral transduction, which make them standard cell lines for CRISPR-Cas9 characterization in eukaryotic cells. These cell lines were purchased directly from the manufacturers but not otherwise authenticated.

Lentiviral production and titering. To package lentivirus, the library plasmid was co-transfected with pMD2.G and psPAX2 into 120 million HEK293FT cells, using Lipofectamine 2000 (Thermo Fisher) in 10 T-225 flasks (Sigma). After $72 \mathrm{~h}$, the supernatant was harvested and concentrated according to the LentiX Concentrator protocol, then stored at $-80^{\circ} \mathrm{C}$. In order to titer this lentiviral preparation, we spinfected 3 million HEK293T cells per well with $0,40,80,120,160$, or $200 \mu$ of the lentiviral supernatant in $2 \mathrm{ml} \mathrm{D} 10$ media supplemented with $8 \mu \mathrm{g} / \mathrm{ml}$ polybrene (Sigma) in 12-well plates (Sigma). The plates were spun at $1000 \times g$ for $2 \mathrm{~h}$ at $37^{\circ} \mathrm{C}$ After spinning, $2 \mathrm{ml}$ fresh D10 media was added to each well and the cells were maintained at $37^{\circ} \mathrm{C}$ for $24 \mathrm{~h}$. The cells were then dissociated with TrypLE, suspended in D10, and counted with a TC20 Automated Cell Counter (Biorad). Then, 2500 cells per well were plated in a black TC-treated 96-well plate with clear well bottoms (Sigma). For each dose, four wells then underwent puromycin selection while four wells continued growth in D10 media. Media was refreshed after $48 \mathrm{~h}$. After $96 \mathrm{~h}$, survival of the selected cells in comparison to the unselected cells was measured by CellTiter-Glo (Promega) and used to calculate the lentivirus MOI. The SaCas9 lentivirus was similarly prepared and titered, using blasticidin selection.

Pairwise library arrayed pilot. As a pilot study, we generated five lentiviral vectors with a shared sgRNA targeting five different target sites, which were mismatched at varied positions to examine specificity. These were synthesized by IDT, cloned into the library lentiviral backbone, and verified by Sanger sequencing. Each construct was individually packaged into lentivirus and titered. HEK293T were transduced by spinfection with the SaCas 9 lentivirus and underwent 6 days of blasticidin selection. Then, wells of 3 million cells each were spinfected with the guide-target lentiviruses, in duplicate, and subjected to puromycin selection for 7 days. Cells were harvested every $24 \mathrm{~h}$. Genomic DNA was extracted and the target sites were sequenced by MiSeq to measure indel rates, using the same computational pipeline as for the pooled screen.

Pairwise library pooled screening workflow. Starting from a library of 88,692 members and $27 \%$ error-free reads in the post-tracrRNA plasmid pool; we spinfected 340 million HEK293T cells with the library lentivirus at a low multiplicity of infection $(\mathrm{MOI}=0.3)$ to achieve a desired representation of $300 \times$. Twenty-four hours after spinfection, we began 5 days of puromycin selection. The cells recovered in antibiotic-free D10 media for 1 day. Then, 340 million cells were harvested for the Day 0 timepoint and 820 million cells were spinfected with the SaCas9 lentivirus at an MOI $=0.4$ to achieve $1000 \times$ representation. The cells recovered in antibiotic-free D10 media for 1 day. They were maintained for 7 days under blasticidin selection and then maintained without further selection for an additional 6 days. The biological replicate of the screen was performed independently on a different day, starting from the initial library spinfection.

Library preparation and sequencing. Genomic DNA was purified from cell pellets of over 340 million cells with a Quick-gDNA Midiprep kit (Zymo), according to the manufacturer's instructions. The library-cassette regions from the entire DNA pool were then PCR amplified with primers designed to target the U6 promoter and the constant sequence downstream of the target site. These primers include Illumina sequencing adapters as extensions (P5 on the forward primer and P7 on the reverse primer) (Supplementary Data 1). There were 12 forward primers 
and 8 reverse primers, which differed in length due to a stagger sequence. The stagger ensures a diversity of base calls in each cycle of sequencing. For each sample, 96 PCRs were performed with: $20 \mu \mathrm{g}$ gDNA, $25 \mu \mathrm{l}$ NEBNext Master Mix (NEB), $0.5 \mu$ l 5 polymerase (NEB), $1 \mu \mathrm{l} \mathrm{DMSO}, 1 \mu \mathrm{l} \mathrm{MgCl}_{2}(25 \mathrm{mM})$, and $0.5 \mu \mathrm{M}$ forward and reverse primers, in $50 \mu \mathrm{l}$ reactions. The thermocycling protocol was: $30 \mathrm{~s}$ at $98^{\circ} \mathrm{C}$, followed by 18 cycles of $98^{\circ} \mathrm{C}$ for $10 \mathrm{~s}, 60^{\circ} \mathrm{C}$ for $30 \mathrm{~s}$, and $72^{\circ} \mathrm{C}$ for $30 \mathrm{~s}$, then a final $5 \mathrm{~min}$ extension at $72{ }^{\circ} \mathrm{C}$. Then, the 96 reactions were pooled. The desired PCR products were then gel extracted and quantified by Qubit. These libraries were subjected to $2 \times 150$ bp paired end sequencing on a HiSeq2500 (Genewiz), with two lanes per sample.

Computational analysis. Indel rates were computed with a Python script ${ }^{27}$. The indel caller discarded reads with substitutions in critical regions, which may arise from sequencing or PCR errors. Error rates were estimated by comparing the constant regions of the library cassette against reference sequences. The pairwise library pooled screen was analyzed with two pipelines. The first pipeline took the library design file and the Day 0 sequencing reads as inputs and outputted a whitelist of validated $\mathrm{rBCs}$, which were associated with error-free library cassettes. The second pipeline took this $\mathrm{rBC}$ list and the Day 3 or 14 sequencing reads as inputs and outputted the indel rate for each guide-target pair, computed from the subset of reads bearing whitelisted $\mathrm{rBCs}$. This process minimizes false positive indels that may arise from mutations in the target site during the synthesis, cloning, or lentiviral packaging. Off:on-target ratios are an indel rate normalized by the indel rate resulting from the perfectly matched guide of equivalent length. We computed this ratio for guide groups with on-target activity $>2 \%$. The script was written in Python and run on an Amazon Web Services EC2 instance. Statistical analyses were performed in Graphpad Prism. Two-sided tests were used in all cases.

GFP reporter of mismatched sgRNA activity. Wild-type and mismatched sgRNA were generated by PCR and transfected as amplicons containing U6 promoter, spacer sequence, and tracrRNA scaffold. Singly mismatched sgRNA were designed by swapping $\mathrm{T}$ and $\mathrm{A}$, or $\mathrm{G}$ and $\mathrm{C}$, at each position. HEK293-GFP were seeded at a density of 100,000 cells/well in 24-well plates. After $24 \mathrm{~h}$, cells were transfected with $250 \mathrm{ng}$ of gRNA amplicon and $750 \mathrm{ng}$ of wild-type SaCas9 plasmid (pAF003). All transfections were performed in duplicate using Lipofectamine 3000 (Life Technologies). At three and a half days post-transfection, cells had media removed and were washed with $0.5 \mathrm{~mL}$ of phosphate-buffered saline (PBS). Two hundred microliters of trypsin was added to the cells and they were incubated at $37^{\circ} \mathrm{C}, 5 \%$ $\mathrm{CO}_{2}$ for $5 \mathrm{~min}$. Trypsinization was halted by adding $0.5 \mathrm{~mL}$ of complete media to each well. Cells were collected and transferred to $1.5 \mathrm{~mL}$ tubes, spun down at $1000 \times g$ for $7 \mathrm{~min}$, washed with $1.0 \mathrm{~mL}$ fluorescence-activated cell sorting (FACS) buffer (PBS with 3\% FBS), spun down again, and resuspended in $200 \mu$ l FACS buffer. Cells were then analyzed with a BD Accuri C6 flow cytometer.

Northern blot. HEK293T cells were transfected with plasmids expressing sgRNA of varying lengths $(18,20,22$, and $24 \mathrm{nt})$ with a U6 promoter. Samples were cotransfected with a SaCas9 plasmid or the corresponding empty vector. After 3 days, sgRNA were purified from cell pellets with a mirVana kit for small RNA isolation (Thermo Fisher). RNAs were heated to $95^{\circ} \mathrm{C}$ for 5 min before loading on $8 \%$ denaturing polyacrylamide gels (SequaGel, National Diagnostics). Afterwards, RNA was transferred to a Hybond N+ membrane (GE Healthcare) and crosslinked with Stratagene UV Crosslinker (Stratagene). Probes were labeled with (gamma$\left.{ }^{32} \mathrm{P}\right)$ ATP (PerkinElmer) with T4 polynucleotide kinase (New England Biolabs). After washing, membrane was exposed to phosphor screen for $1 \mathrm{~h}$ and scanned with a phosphorimager (Typhoon). The sgRNA expression ratio was quantified based on the intensity of the bands in the image using ImageJ analysis software.

Model of SaCas9 specificity. Parameter values for the nonlinear model were derived using Hamiltonian Monte Carlo sampling as implemented in the Rstan package. The default No U-Turn Sampler was used to draw 1500 samples across eight independent chains, with the first 500 samples in each chain being discarded as part of the warmup phase. Convergence of the posterior distributions for the parameters was verified by calculating the R-hat statistic and verifying that values for model parameters were $<1.1$.

The statistical model is implemented as follows. The indel rate $I_{j M}$ observed for a guide $j$ targeting a sequence with mismatches $M$ is calculated by assuming that the on-target activity of the guide $g_{j}$ is decreased by additive penalties incurred for each mismatch $\left(\Delta \Delta G_{k}\right)$ and that the calculated indel rate is subject to independent Gaussian errors $\varepsilon_{j, M}$.

$$
I_{j, M}=g_{j} \exp \left(\sum_{k \in M}-\Delta \Delta G_{k}\right)+\epsilon_{j, M}
$$

The penalty terms $\Delta \Delta G_{k}$ are restricted to being non-negative and regularized by enforcing a prior distribution that is exponential with mean $\beta=1$. The error terms $\varepsilon_{j, M}$ are normally distributed with mean 0 and standard deviation 0.1 (as estimated from comparisons across replicates). For ease of interpretation, the same model can be reparametrized so that the penalties are multiplicative, as follows:

$$
I_{j, M}=g_{j} \prod_{k \in M} f(\operatorname{pos}(k), \operatorname{type}(k))+\epsilon_{j, M}
$$

In this case, the penalty on guide activity as a function of the mismatch position and type can be represented as a value between 0 and 1 . Parameter values were independently derived for each guide length by separating the data into disjoint training sets, leading to a separate mismatch effect matrix $f_{L}$ for each value of guide length $L$ assayed.

This score fit the observations well for most guides in our dataset, except for guides with very low on-target activity (Supplementary Fig. 5B, C). This may be explained by a low signal-to-noise ratio for these ineffective guides.

Code availability. The code for the analysis, modeling, and specificity score are available on the Editas Medicine Github (https://github.com/editasmedicinedev/ pairwise-library-screen). They are available under the BSD 3-Clause Clear License.

Data availability. The datasets generated during the current study are available in the NCBI SRA repository (SRP147992).

Received: 9 February 2018 Accepted: 3 July 2018

Published online: 27 July 2018

\section{References}

1. Ran, F. A. et al. In vivo genome editing using Staphylococcus aureus Cas9. Nature 520, 186-191 (2015).

2. Friedland, A. E. et al. Characterization of Staphylococcus aureus Cas9: a smaller Cas9 for all-in-one adeno-associated virus delivery and paired nickase applications. Genome Biol. 16, 257 (2015).

3. Tabebordbar, M. et al. In vivo gene editing in dystrophic mouse muscle and muscle stem cells. Science 351, 407-411 (2016).

4. Nelson, C. E. et al. In vivo genome editing improves muscle function in a mouse model of Duchenne muscular dystrophy. Science 351, 403-407 (2016)

5. Yang, Y. et al. A dual AAV system enables the Cas9-mediated correction of a metabolic liver disease in newborn mice. Nat. Biotechnol. 34, 334-338 (2016).

6. Yin, C. et al. In vivo excision of HIV-1 provirus by saCas9 and multiplex single-guide RNAs in animal models.Mol. Ther. 25, 1168-1186 (2017).

7. Kleinstiver, B. P. et al. Broadening the targeting range of Staphylococcus aureus CRISPR-Cas9 by modifying PAM recognition. Nat. Biotechnol. 33, 1293-1298 (2015).

8. Slaymaker, I. M. et al. Rationally engineered Cas9 nucleases with improved specificity. Science 351, 84-88 (2016).

9. Chen, B. et al. Expanding the CRISPR imaging toolset with Staphylococcus aureus Cas9 for simultaneous imaging of multiple genomic loci. Nucleic Acids Res. 44, e75 (2016).

10. Bystrykh, L. V. Generalized DNA barcode design based on Hamming codes. PLoS ONE 7, e36852 (2012).

11. Tsai, S. Q. et al. GUIDE-seq enables genome-wide profiling of off-target cleavage by CRISPR-Cas nucleases. Nat. Biotechnol. 33, 187-197 (2015).

12. Fu, Y., Sander, J. D., Reyon, D., Cascio, V. M. \& Joung, J. K. Improving CRISPR-Cas nuclease specificity using truncated guide RNAs. Nat. Biotechnol. 32, 279-284 (2014).

13. Morgens, D. W. et al. Genome-scale measurement of off-target activity using Cas9 toxicity in high-throughput screens. Nat. Commun. 8, 15178 (2017).

14. Wyvekens, N., Topkar, V. V., Khayter, C., Joung, J. K. \& Tsai, S. Q. Dimeric CRISPR RNA-guided FokI-dCas9 nucleases directed by truncated gRNAs for highly specific genome editing. Hum. Gene Ther. 26, 425-431 (2015).

15. Xu, X., Duan, D. \& Chen, S. J. CRISPR-Cas 9 cleavage efficiency correlates strongly with target-sgRNA folding stability: from physical mechanism to offtarget assessment. Sci. Rep. 7, 143 (2017).

16. Kim, D. et al. Digenome-seq: genome-wide profiling of CRISPR-Cas9 offtarget effects in human cells. Nat. Methods 12, 237-243 (2015).

17. Kim, D. et al. Genome-wide analysis reveals specificities of Cpf1 endonucleases in human cells. Nat. Biotechnol. 34, 863-868 (2016).

18. Kim, D., Kim, S., Kim, S., Park, J. \& Kim, J. S. Genome-wide target specificities of CRISPR-Cas9 nucleases revealed by multiplex Digenome-seq. Genome Res 26, 406-415 (2016).

19. Tsai, S. Q. et al. CIRCLE-seq: a highly sensitive in vitro screen for genomewide CRISPR-Cas9 nuclease off-targets. Nat. Methods 14, 607-614 (2017).

20. Hsu, P. D. et al. DNA targeting specificity of RNA-guided Cas9 nucleases. Nat. Biotechnol. 31, 827-832 (2013).

21. Fu, B. X., Hansen, L. L., Artiles, K. L., Nonet, M. L. \& Fire, A. Z. Landscape of target: guide homology effects on Cas9-mediated cleavage. Nucleic Acids Res 42, 13778-13787 (2014). 
22. Pattanayak, V. et al. High-throughput profiling of off-target DNA cleavage reveals RNA-programmed Cas9 nuclease specificity. Nat. Biotechnol. 31, 839-843 (2013).

23. Fu, B. X., St Onge, R. P., Fire, A. Z. \& Smith, J. D. Distinct patterns of Cas9 mismatch tolerance in vitro and in vivo. Nucleic Acids Res. 44, 5365-5377 (2016).

24. Doench, J. G. et al. Optimized sgRNA design to maximize activity and minimize off-target effects of CRISPR-Cas9. Nat. Biotechnol. 34, 184-191 (2016).

25. Josephs, E. A. et al. Structure and specificity of the RNA-guided endonuclease Cas9 during DNA interrogation, target binding and cleavage. Nucleic Acids Res. 44, 2474 (2016).

26. Jafari, N. et al. CRISPR-Cas9 mediated NOX4 knockout inhibits cell proliferation and invasion in HeLa cells. PLoS ONE 12, e0170327 (2017).

27. Ran, F. A. et al. Double nicking by RNA-guided CRISPR Cas9 for enhanced genome editing specificity. Cell 154, 1380-1389 (2013).

\section{Acknowledgements}

We would like to thank Team Editas for helpful discussions and support.

\section{Author contributions}

P.D.H. devised the concept. J.T., J.S.G., and P.D.H. designed the library with input from O.O.A. J.T., N.H., and P.D.H. carried out screen. A.E.F. performed G.F.P. knockout validation. X.W. performed Northern blots. J.S.G. contributed indel detection code. J.T. and P.D.H. wrote screen analysis code and performed data analysis. L.A.B. developed the specificity score. J.T., L.A.B., V.E.M., C.J.W., and P.D.H. interpreted results and wrote the manuscript with help from all authors.

\section{Additional information}

Supplementary Information accompanies this paper at https://doi.org/10.1038/s41467018-05391-2.

Competing interests: J.T., L.A.B., N.H., A.E.F., J.S.G., V.E.M., C.J.W., and P.D.H. are employees, consultants, or former employees of Editas Medicine. X.W. and O.O.A. declare no competing interests.

Reprints and permission information is available online at http://npg.nature.com/ reprintsandpermissions/

Publisher's note: Springer Nature remains neutral with regard to jurisdictional claims in published maps and institutional affiliations.

(c) (i) Open Access This article is licensed under a Creative Commons Attribution 4.0 International License, which permits use, sharing, adaptation, distribution and reproduction in any medium or format, as long as you give appropriate credit to the original author(s) and the source, provide a link to the Creative Commons license, and indicate if changes were made. The images or other third party material in this article are included in the article's Creative Commons license, unless indicated otherwise in a credit line to the material. If material is not included in the article's Creative Commons license and your intended use is not permitted by statutory regulation or exceeds the permitted use, you will need to obtain permission directly from the copyright holder. To view a copy of this license, visit http://creativecommons.org/ licenses/by/4.0/.

(C) The Author(s) 2018 